\title{
INTERNET EN EL AULA: A LA CAZA DEL TESORO
}

\author{
Jordi Adell \\ Centre d'Educació i Noves Tecnologies \\ Universitat Jaume I \\ jordi@uji.es
}

\section{RESUMEN:}

Una caza del tesoro es un tipo de actividad didáctica muy sencilla que utilizan los docentes que integran la Internet en el currículum. Consiste en una serie de preguntas y una lista de direcciones de páginas web de las que pueden extraerse o inferirse las respuestas. Algunas incluyen una "gran pregunta" al final, que requiere que los alumnos integren los conocimientos adquiridas en el proceso En este texto se explica qué es una caza del tesoro, sus potencialidades didácticas, dónde encontrar cazas ya preparadas en la Internet y cómo prepararlas nosotros mismos, en función de nuestros objetivos curriculares.

\section{INTRODUCCIÓN}

Una "caza del tesoro" (en inglés "Treasure Hunt", "Scavenger Hunt" o "Knowledge Hunt", ya que de las tres formas se la conoce) es una de las estructuras de actividad didáctica más populares entre los docentes que utilizan la Internet en sus clases. En esencia, una caza del tesoro es una hola de trabajo o una página web con una serie de preguntas y una lista de páginas web en las los alumnos buscan las respuestas. Al final se suele incluir la "gran pregunta", cuya respuesta no aparece directamente en las páginas web visitadas y que exige integrar y valorar lo aprendido durante la búsqueda. Las cazas del tesoro son estrategias útiles para adquirir información sobre un tema determinado y practicar habilidades y procedimientos relacionados con las tecnologías de la información y la comunicación en general y con el acceso a la información a través de la Internet en particular. 


\section{Un par de ejemplos:}

La siguiente caza del tesoro es muy simple: todas las respuestas están en el mismo sitio web (prácticamente una lista con las respuestas) y no hay una gran pregunta final. Su objetivo es adquirir información sobre la naturaleza de la luz. Puede utilizarse como actividad complementaria en una unidad didáctica sobre dicho tema.

LOS COLORES DEL UNIVERSO:

http://www.education-world.com/a lesson/hunt/hunt001.shtml

\section{Instrucciones:}

¿Qué sabes sobre los colores del universo? Utiliza la información que encontrarás en el sitio web de más abajo para responder las preguntas de esta página. Escribe las respuestas en las líneas que hay debajo de cada pregunta.

\section{Recurso Web:}

Why Is the Sky Blue? (¿Por qué el cielo es azul?) URL: http://www.why-is-the-sky-blue.org

Preguntas:

1. ¿De qué color es la onda más larga de luz?

2. ¿Qué clase de partículas atmosféricas hacen que Marte sea rojo?

3. ¿De qué color es el océano en un día nublado?

4. ¿Qué colores hay en la luz blanca?

5. ¿Qué clase de partículas se encuentran en la atmósfera de la Tierra?

Bien, aquí van las respuestas: la luz roja es la que tiene la longitud de onda más larga; Marte es rojo debido a las partículas de hierro que hay en suspensión en su atmósfera; en un dia nublado, el oceano aparece gris; la luz blanca está formada por todos los otros colores del espectro; y la atmósfera de la Tierra contiene nitrógeno, oxígeno, partículas de polución y agua.

El siguiente ejemplo de caza del tesoro, titulada Murciélagos, en cambio, es una actividad más compleja cuyo objetivo es que los alumnos adquieran la mayor cantidad posible de información sobre los murciélagos. La pregunta final, además, es un buen ejemplo de cómo redondear una actividad de este tipo estimulando la reflexión sobre lo aprendido. 




Introducción:

¿Has visto alguna vez un murciélago? Seguro que sí. ¿Sabes que viven prácticamente en todas las regiones del mundo y que hay de muchos tipos? En esta actividad vamos a aprender algunas cosas sobre los murciélagos y sus costumbres. Como verás, son unos animales muy interesantes sobre los que la gente, a veces, tiene ideas equivocadas.

\section{Preguntas:}

1. ¿A qué especie animal pertenecen los murciélagos? ¿Cuáles son sus características?

2. ¿Cuántas especies de murciélagos hay en la península Ibérica? ¿Y en las Islas Canarias? Copia y pega tres fotos o dibujos de diferentes especies de murciélagos en tu cuaderno.

3. ¿Qué tipo de murciélago se encuentra en peligro de extinción en la Comunidad Valenciana? ¿Cómo es su nombre científico? ¿Cómo se llama en valenciano?

4. ¿Qué comen los murciélagos?

5. ¿Cuando salen los murciélagos (estación del año, hora del día, lugares en los que se les ve, etc.)?

6. ¿En qué consiste la ecolocación? Define los cuatro tipos que existen.

7. ¿Qué es la econavegación de los murciélagos? ¿Qué tipo de murciélagos la realizan? Explica brevemente las características de estos murciélagos.

8. ¿Son completamente ciegos los murciélagos?

9. ¿En qué tipo de películas aparecen los murciélagos? ¿Por qué crees que los murciélagos aparecen en estas películas?

\section{Recursos:}

- Mamiferos: http://members.es.tripod.de/valdavia/mamiferos.htm

- K8AIT Principios de la Aeronáutica. Animales: http://wings.avkids.com/Libro/Animals/advanced/bats-01.html

- Los Murciélagos Ecología e Historia Natural http://www.acguanacaste.ac.cr/rothschildia/v4n1/textos/murcielagos.html 
- Fauna Ibérica: http://www.faunaiberica.org/especies.php3?esp=73

- Revista Quercus: Situación actual de los murciélagos en la península Ibérica: http://www.quercus.es/RedQuercus/Articulos/murci.htm

- Mitos y realidades de los murciélagos: http://www.uanl.mx/publicaciones/vidauni/38/murcielagos.html

- Internatura: http://www2.uji.es/cyes/internatura/estudios/listas/Imamif.html

La gran pregunta:

Ahora, piensa en todo lo que has aprendido sobre los murciélagos e intenta contestar a esta pregunta: ¿Por qué los murciélagos son beneficiosos para los seres humanos?

\section{CONCEPTOS, PROCEDIMIENTOS Y ACTITUDES}

Una caza del tesoro bien diseñada va más allá de la adquisición de pequeñas unidades de información, más o menos estructurada, sobre un tema determinado. March (1999) sugiere que escogiendo adecuadamente preguntas que definan las dimensiones fundamentales de un tema, los alumnos no sólo averiguan respuestas concretas, sino que profundizan en los aspectos esenciales del tema. Además del aprendizaje de hechos y conceptos, una caza del tesoro estimula la adquisición de destrezas sobre tecnología de la información y comunicación, conocimientos prácticos sobre Internet, la web y la navegación por la información online.

Luzón (2001), que propone su utilización en la enseñanza de inglés como segunda lengua (ESL) con estudiantes universitarios, resume los puntos fuertes de las cazas en los siguientes tres aspectos:

a) Promueven la adquisición de conocimientos sobre un tema,

b) Desarrollan destrezas de búsqueda de información en la web; y

c) Mejoran las destrezas de lectura y comprensión de textos.

En la enseñanza de ESL, por ejemplo, facilitan que los estudiantes entren en contacto con el lenguaje auténtico, relacionado con su disciplina, que hay en la red, animan a la lectura orientada a una finalidad específica, aumentan el conocimiento de vocabulario genérico y especializado, consolidan el lenguaje previamente aprendido $y$, además, ayudan a los estudiantes a desarrollar la habilidad de realizar inferencias y predecir el contexto de un texto (Luzón, 2001). Es más, las capacidades hipertextuales de la web permiten que los estudiantes sigan sus propios "caminos" y exploren lugares relacionados con las páginas iniciales propuestas para la exploración en los que pueden encontrar más información sobre el mismo tema.

Las cazas del tesoro pueden diseñarse para elicitar destrezas básicas de pensamiento como comprensión y construcción de conocimientos. Pare ello se incluyen preguntas que procuren 
que los alumnos utilicen la información de las páginas web para describir o esquematizar su contenido, crear listas, resumir y tomar notas, etc. También pueden diseñarse para que los alumnos desarrollen destrezas de alto nivel como la aplicación y el análisis del conocimiento. Para ello se procura que los alumnos organicen y categoricen la información, la analicen y la interpreten, la discutan, la expliquen, la comparen, realicen generalizaciones, etc. (Hamilton, 1999).

Las materia prima para desarrollar ambos tipos de destrezas es la misma: información en formatos diversos, aunque su profundidad y complejidad variará en función de la edad de los alumnos y de los objetivos perseguidos. Es el proceso, es decir, lo que intentamos que los alumnos hagan con la información, lo que cambiará en función de los objetivos, la edad y capacidad de los alumnos. Las preguntas son nuestra herramienta para moldear dicho proceso. Una buena caza del tesoro es la conjunción de preguntas adecuadamente formuladas y recursos web con contenidos interesantes, bien conectados con el currículum y adecuados al nivel de nuestros alumnos.

Las "cazas del tesoro" se han hecho muy populares entre los docentes como estructura didáctica para la integración por varias razones:

1. Son relativamente fáciles de crear para el docente y son divertidas y formativas para los estudiantes. Bien planteadas, parecen un juego, pero los alumnos aprenden muchas cosas durante una caza del tesoro (y no sólo las respuestas a las preguntas).

2. Pueden tratar sobre casi cualquier aspecto del currículum (siempre que encontremos recursos adecuados al tema y edad de los alumnos en la Internet) y proporcionan conocimientos sobre los contenidos y experiencia en el manejo de herramientas Internet.

3. Pueden utilizarse como actividades para realizar en grupo o individualmente.

4. Pueden ser simples o complicadas, tal como dicten las circunstancias. A los alumnos más pequeños se les pueden proponer menos preguntas, con formulaciones más simples y con los vínculos necesarios para resolverlas a continuación de cada pregunta. Los mayores pueden recibir sólo un tema amplio y se les pide que encuentren por sí mismos la información en un solo sitio, más amplio y complejo, o que busquen sus propias fuentes para obtener la información necesaria o se les proporciona un punto de entrada a una serie de lugares relacionados.

Buscar información es una destreza importante para los estudiantes. La Internet constituye una excelente herramienta para aprender de modo independiente o para obtener materiales de todo tipo para realizar las tareas escolares. Pero es importante enseñar no sólo a buscar, sino también a evaluar la fiabilidad y exactitud de la información encontrada. La Internet contiene enormes cantidades de información interesante, útil y veraz. Pero no es una enciclopedia (March, 1999). A diferencia de una enciclopedia, la web no está organizada lógicamente, no hay un único criterio o perspectiva, sino múltiples y contradictorios, no posee un nivel de profundidad uniforme en el tratamiento de los temas, no está estructurada coherentemente y muchos de sus contenidos son perfectamente prescindibles. A pesar de ello, podemos encontrar auténticas joyas sobre los temas más variados. Como criterio general, los docentes deberían buscar en la red aquello de lo que no disponen en el aula o en el centro escolar. 
Una alternativa para estudiantes de niveles superiores es hacer que, en lugar de resolverlas, preparen ellos mismos sus propias "cazas del tesoro" adoptando el papel de profesores. Las cazas pueden prepararse en equipo y, posteriormente, cada grupo puede resolver la caza elaborada por otro equipo. En este caso, los criterios para evaluar la calidad de la caza deberían incluir la representatividad, pertinencia o relevancia de las preguntas al tema en cuestión y a los recursos disponibles.

\section{DÓNDE ENCONTRAR CAZAS DEL TESORO EN INTERNET}

Hay multitud de sitios web con colecciones de cazas del tesoro hechas por maestros y maestras de todos los niveles. De hecho, basta con introducir en cualquier buscador tipo Google la cadena "treasure hunt" o "scavenger hunt" o "knowledge hunt" para encontrar, literalmente, miles de ellas. A continuación incluimos algunas páginas que contienen colecciones de cazas del tesoro.

- Internet Scavenger Hunts (University of Tennessee at Chattanooga): http://cecasun.utc.edu/ tpa/mcallister/scavenger.html

- The Scavenger Hunt Page (Apple Valley (lowa) Area Education Agency 14): http://www.aea14.k12.ia.us/technology/ScavengerHunt.html

- Internet-Based Scavenger Hunts for Students: http://www.ctnba.org/ctn/k8/treasure.html

Aunque en la Internet podemos encontrar miles de cazas del tesoro ya preparadas, es conveniente que diseñemos nuestras propias cazas. Hay un par de factores que es necesario aconsiderar. El primero es la volatilidad de los recursos web. Aquella página tan interesante que encontramos hace unos meses, puede que mañana, cuando nuestros alumnos intenten entrar en ella, haya desaparecido. El aula no es el lugar idóneo para descubrir que la mitad de nuestros enlaces no conducen a ningún sitio. Por tanto, para utilizar cazas del tesoro preparadas por otros docentes es necesario comprobarlas exhaustivamente poco antes de ponerlas en marcha. Pese a todo, compartirlas con otros docentes mediante la Internet es una buena idea ya que proporcionan ideas y lugares interesantes que facilitan mucho el trabajo de preparar cazas.

El segundo inconveniente es de mayor calado: su adecuación a nuestro currículum, objetivos y alumnos. Una buena caza del tesoro debe diseñarse teniendo en cuenta los objetivos que perseguimos, lo que ya saben nuestros alumnos, sus habilidades en el manejo de la tecnología, el contexto escolar, el currículum, la dinámica de la clase, los medios tecnológicos disponibles en el centro, etc. ¿Quién conoce a la perfección todos estos pormenores? Evidentemente, cada maestro y maestra. La conclusión es obvia: diseñemos nuestras propias cazas del tesoro, aunque utilicemos ideas y sitios web que hemos encontrado en otras, porque serán mucho mejores (para nuestro contexto) que las que podamos encontrar por la red. 


\section{CÓMO PREPARAR NUESTRAS PROPIAS CAZAS DEL TESORO}

El procedimiento de diseño y desarrollo de una "caza del tesoro" puede resumirse en los siguientes pasos:

\section{Elección del tema y de los objetivos didácticos.}

Sería conveniente escoger algún tema respecto al cual estemos insatisfechos con nuestro trabajo actual (si algo ya nos sale bien, ¿para qué cambiarlo?) y que intentemos crear algunas actividades nuevas, como cazas del tesoro, para cambiar la dinámica de la clase, nuestro enfoque o las actividades de los alumnos. Podemos añadir la caza a una unidad didáctica que ya tengamos elaborada y a la que queramos incluir el uso de la Internet como un recurso más.

Escojamos los objetivos del currículum que queremos conseguir con la caza. Incluyamos criterios de evaluación para saber si se han conseguido o no.

\section{Preparación de la hoja de trabajo.}

Podemos preparar una hoja de trabajo en soporte papel o en HTML para que los alumnos la consulten online o en el disco duro del ordenador y puedan ir a los recursos web con un solo clic de ratón, sin necesidad de escribir los URLS.

Estructuraremos la hoja de trabajo en cuatro partes: Introducción, Preguntas, Recursos y "La gran pregunta"

\subsection{Introducción}

En la Introducción se deben describir la tarea y las instrucciones para realizarla. Ante todo debemos despertar el interés de los alumnos por averiguar las respuestas a las preguntas.

\subsection{Preguntas}

Debemos listar y numerar las preguntas o pequeñas actividades a realizar. Para formular las preguntas, en el caso de alumnos jóvenes, deberíamos copiar y pegar las frases exactas que contienen la información relevante que queremos que encuentren (una de cada página web de la lista de recursos) y luego transformarlas en preguntas directas. Para alumnos más mayores, se pueden efectuar preguntas que impliquen actividades más complejar, relacionadas con la lectura comprensiva, la inferencia, la reunión y organización de información, la comparación, etc. Si plantamos prequeñas actividades, deben poderse realizar con cierta rapidez: leer un mapa, efectuar un cálculo, averiguar el resultado de una pequeña simulación, etc. 
Por ejemplo, se puede preguntar sobre el por qué(análisis: ¿por qué las cosas ocurren de la manera en que lo hacen?), sobre el cómo (solución de problemas: ¿cómo pueden hacerse mejor?) y sobre el qué hacer (toma de decisiones: ¿qué opción debo escoger?). Para hacer preguntas consistentes, primero se debe escribir la pregunta, luego reformularla (un máximo de 50 palabras) especificando qué pretende que aprendan los alumnos respondiéndola. Luego, hay que revisar la primera formulación.

\subsection{Recursos}

A continuación debemos listar los títulos y URLs de las páginas a consultar para encontrar las respuestas a las preguntas o realizar las actividades. Se pueden utilizar buscadores especializados o genéricos o herramientas ad hoc como Trackstar (http://trackstar.hprtec.org). También se pueden utilizar o adaptar una "caza del tesoro" de las que existen en la Internet y que han sido elaboradas y probadas por profesores.

Un buen hábito es marcar los recursos interesantes que encontremos cuando navegamos por la red, aún cuando no sean lo que estábamos buscando: así podremos explorarlos posteriormente.

Servicios como TrackStar "acumulan sabiduría" de una enorme cantidad de maestros y maestras en forma de listas de links sobre un tema determinado. Los portales educativos tienen colecciones de recursos de interés curricular organizados por temas. Por ejemplo, Education Planet (http://www.education-planet.com) oEducation World (http://www.educationworld.com). En castellano pueden encontrarse recursos en las web de la XTEC (http://www.xtec.es/), el Centro Nacional de Información y Comunicación Educativa (CNICE) (http://www.cnice.mecd.es/), en Profes.net y en portales educativos o buscadores generalistas como Yahoo!, Google o Altavista. No es necesario decir que la cantidad de recursos educativos en inglés es muy superior a la disponible en otros idiomas. Pero hay multitud de sitios de interés: periódicos, museos, universidades, editoriales, portales educativos, consejerías de educación, planes y proyectos de nuevas tecnologías, redes educativas, páginas personales de docentes con colecciones de links interesantes, etc.

Es recomendable hacer preguntas no sólo sobre el qué, sino también sobre el por qué (implica análisis: ¿por qué las cosas ocurren de determinada manera?), sobre el cómo (implica solución de problemas: ¿cómo pueden hacerse mejor?, por ejemplo) y sobre el qué hacer (implica toma de decisiones: ¿qué opción debo escoger?). Para hacer preguntas consistentes, podemos hacerlo de la siguiente manera: primero escribimos la pregunta, luego la reformulamos (un máximo de 50 palabras) especificando qué se pretende que aprendan los alumnos respondiéndola. Luego, a la luz de nuestros objetivos, revisamos la primera formulación.

\subsection{La gran pregunta}

Incluir una pregunta final, global, cuya respuesta no se encuentre directamente en ninguna página de la lista de recursos, sino que dependa de las respuestas a las preguntas anteriores y de lo aprendido buscando las respuestas. Idealmente, debería coincidir con un objetivo curricular y puede incluir aspectos valorativos y de opinión personal sobre el tema buscado. Aquí podríamos trabajar normas, actitudes y valores pidiendo que los alumnos reflexionaran 
sobre las implicaciones personales, sociales, políticas, etc. del tema de la caza (si se presta a ello, naturalmente).

\section{Formación de grupos}

Se pueden formar grupos con los estudiantes en función de su experiencia y conocimientos sobre la Internet y la Web, de modo que los más experimentados ayuden y enseñen a los menos experimentados. Debemos asegurarnos de que los menos familiarizados tengan oportunidad de adquirir la experiencia que les falta (que no sean "apartados" del ordenador por sus compañeros o se inhiban ellos mismos). No debemos convertir la caza en una competición en base al tiempo.

\section{Procedimiento}

Dependiendo del número de ordenadores conectados a la Internet que dispongamos, organizaremos los periodos de búsqueda y los de organización de la información y respuesta a la preguntas. En un aula informática, todos los grupos pueden disponer de un ordenador y espacio para trabajar ( 2 o 3 por ordenador). En un aula, con un rincón del ordenador y uno o varios ordenadores, los niños y niñas irán turnándose ante la máquina y, posteriormente, elaborando las respuestas.

\section{ALGUNOS CONSEJOS ÚTILES PARA DISEÑAR CAZAS DEL TESORO:}

1. Identificar claramente el tema y el área de la caza (esto ayudará posteriormente, sobre todo si la publicamos y la compartimos con otros docentes por Internet).

2. Se debe procurar que las preguntas provoquen el pensamiento y la reflexión. Los estudiantes no deben limitarse a "copiar y pegar", deben pensar para responder.

3. Menos es más. Nunca deberíamos incluir más de diez vínculos. Con niños pequeños, lo ideal es uno o dos.

4. Se debe fijar un límite de tiempo para la caza del tesoro y monitorizar qué hacen los estudiantes: si se atascan con el navegador, si se distraen, si terminan muy rápidamente y sus respuestas iniciales son superficiales, si han entendido la tarea, etc.

5. Se debe planificar cómo compartirán la información los alumnos; una hoja de trabajo a mano o algún otro tipo de producto (una presentación, un texto electrónico, etc.).

6. Finalmente, se debe comprobar si hay vínculos muertos: la web es muy volátil y lo que un día está, al siguiente ha cambiado.

\section{EVALUACIÓN}

La manera más sencilla de evaluar una caza es en función del producto: es decir, de la cantidad y calidad de los aciertos de los estudiantes. Sin embargo, es interesante establecer algunos indicadores de la calidad del proceso: grado de elaboración de las estrategias de búsqueda, originalidad, trabajo en equipo, manejo de la tecnología, etc. 
Una práctica recomendable es corregir la caza entre todos y dar oportunidad de que aquellos alumnos que no han encontrado las respuestas o las han elaborado poco, la rehagan con la colaboración del maestro o maestra.

\section{REFERENCIAS:}

Hamilton, Sondra M. (1999). Scavenger Hunts. Online. Consultado 1/04/03: http://www.cybercom.net/ iham/beagle/pages/scavenger hunt.htm

Lewis, Beth (s.f.). How to Make Online Scavenger Hunts. Online, consultado: 1/04/2003. http://k-

6educators.about.com/c/ht/00/07/How Online Scavenger Hunts0962932801.htm? once=true\&iam=metacrawl\&terms $=+$ webquest.

Luzón, MJ: (2001). Information Collection and Analysis Activities: The Treasure Hunt. Teaching English with Technology, 1(4), Online. Consultado 27/8/2001 http://www.iatefl.org.pl/sig/call/i_esp4.htm.

March (1999). The Six Web-and-Flow Activity Formats. Online. Consultado: 27/8/2001. http://www.web-and-flow.com/help/formats.asp

Sailaja Athyala (2001). Scavenger Hunt. Online, Consultado: 1/4/03. http://www.hc.cc.tx.us/titlev/TitleVFacultyTraining/workshops/Scavenger \%20Hunt.pdf.

Starr, Linda (1999). Scavenger Hunts: Searching for Treasure on the Internet!, Education World. Online, consultado $10 / 8 / 01$ http://www.educationworld.com/a curr/curr113.shtml.

\section{Para citar este artículo:}

Adell, J. (2003). Internet en el aula: A la caza del tesoro. EDUTEC, Revista Electrónica de Tecnología Educativa, 16. Recuperado el dd/mm/aa de http://edutec.rediris.es/Revelec2/Revelec16/Adell.html 